\title{
TV/Series
}

1 | 2012

Les Séries télévisées américaines contemporaines :

entre la fiction, les faits, et le réel

\section{Mad Men et l'art de la mise en perspective}

\section{Lionel Dufaye}

\section{(2) OpenEdition}

Journals

Édition électronique

URL : http://journals.openedition.org/tvseries/1083

DOI : 10.4000/tvseries.1083

ISSN : 2266-0909

Éditeur

GRIC - Groupe de recherche Identités et Cultures

Référence électronique

Lionel Dufaye, « Mad Men et l'art de la mise en perspective », TV/Series [En ligne], 1 | 2012, mis en ligne le 15 mai 2012, consulté le 01 mai 2019. URL : http://journals.openedition.org/tvseries/1083 ; DOI :

$10.4000 /$ tvseries. 1083

\section{(c) $\oplus \Theta \Theta$}

TV/Series est mis à disposition selon les termes de la licence Creative Commons Attribution - Pas d'Utilisation Commerciale - Pas de Modification 4.0 International. 
Le présent article propose une analyse de la saison 1 de Mad Men sous l'angle de l'altérité. L'objectif est de montrer que les grandes composantes de la série, qu'il s'agisse des thématiques, du cadre temporel, des personnages ou encore de leurs dimensions relationnelles, se définissent tout autant par ce qu'elles sont que par ce qu'elles ne sont pas. Or, ces références en négatif sont mises en scène dans la série, parfois de manière relativement explicite, parfois de manière beaucoup plus oblique. C'est sur quelques-unes de ces références externes que nous nous arrêterons pour montrer de quelle manière elles contribuent pleinement de la mise en place des réseaux de sens de la série. Ainsi, après quelques remarques sur le graphisme et la logographie du générique, nous nous pencherons sur des personnages clés pour comprendre comment ils sont définis tout autant par des propriétés intrinsèques (pour certaines empruntées directement à des personnages extra-fictionnels) que par leur mode de relation avec d'autres personnages.

\title{
A Twilight Zone.
}

\author{
You better start swimmin \\ Or you'll sink like a stone \\ For the times they are a-changin'. \\ Bob Dylan, 1964 .
}

$\mathrm{L}$ a première saison de Mad Men ne se passe pas dans les années 60. Elle se passe en 1960. A la charnière de deux époques, au matin d'une décennie qui va être le théâtre de bouleversements sociaux et culturels dont les effets résonnent jusqu'à aujourd'hui : mouvements contestataires, poussées émancipatrices, explosion de l'hyper-consommation, amplification de la culture pop et télévisuelle, valorisation de la pensée subversive... D’une certaine manière, Mad Men est la scénarisation d'une collision entre deux sociétés en rupture, que l'on va observer tel un Janus en stéréoscopie, un regard vers l'avenir, l'autre dans le rétroviseur. Mad Men est le sauve-qui-peut d'une génération qui, toute lovée dans la croissance compensatrice de son après-guerre, est tirée de son sommeil par les métamorphoses d'un monde qu'elle découvre en brutale mutation. La chute cauchemardesque du générique est l'indice proleptique de ce vertige face à une société qui change de mains, et dont les repères se dérobent.

Si cette altérité constitue le cœur fondateur de la série, elle affleure en réalité à tout un ensemble de niveaux de représentation, allant de la construction de doubles à des oppositions thématiques en 
passant par des mises en perspective historiques ou encore des choix de formalisation. Ces différentes manifestations de l'altérité, qui ne sont que les réverbérations d'un socle thématique, sont sans doute trop nombreuses pour être analysées de manière exhaustive, mais l'on peut néanmoins ouvrir la porte sur quelques-unes d'entre elles à titre d'illustration. C'est ce que se propose de faire cet article ${ }^{1}$.

\section{Des couleurs éteintes aux couleurs et teintes.}

The fifties were captured in black and white, most often by still photographers; by contrast, the decade that followed was, more often than not, caught in living color on tape or film.

D. Halberstam, 1993, The Fifties.

Les années 50, largement « encrées » dans notre mémoire collective en noir et blanc, voient avec Mad Men leurs couleurs ressuscitées comme sous l'effet d'un défibrillateur. Peut-être est-ce de cette façon que nous pouvons interpréter l'intervention du rouge dans la logographie du titre ; un monde en noir et blanc duquel surgit une polychromie brutale qui signale qu'il s'agit moins de pasticher une époque que de la rendre sous « ses vraies couleurs ».
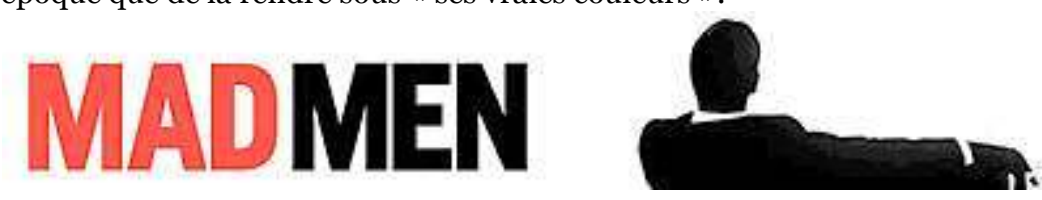

En soi, la couleur établit ainsi une distance par rapport à la représentation bichromique standard de l'époque qu'elle dépeint; époque largement idéalisée, notamment par le biais du cinéma, de la photo, mais aussi de la publicité.

Avant de revenir sur ce point, arrêtons-nous un instant sur le logo de Mad Men, qui de par sa typographie en majuscules très fonctionnelles et sa composition de « couleurs » basiques, se présente telle une logographie de marque, où des valeurs de branding entrent déjà en résonnance avec la thématique publicitaire. En termes d'accroche, on peut dire que le marketing de la série se confond d'emblée avec les techniques qu'elle scénarise

L'utilisation du trio noir-blanc-rouge a, on s'en rend intuitivement compte, une dynamique particulière :

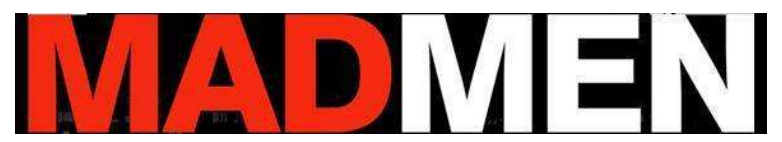

${ }^{1}$ Il est important de souligner que cet article ne fait référence qu'à la saison 1 de $\mathrm{Mad}$ Men. 
Les valeurs noir / blanc fixent les limites polaires de notre champ de sensations, au sein duquel intervient un rouge saturé, teinte la plus forte en énergie de photons perceptible par l'œil humain, et qui impose de ce fait une force d'attraction sur le regard.

Sans doute cela explique-t-il pourquoi, ce code couleur, relativement omniprésent dans la logographie contemporaine, est associé à toute une tradition militante et revendicatrice.

Ainsi, on citera le cas du constructivisme bolchévique qui a largement exploité cette association de valeur :

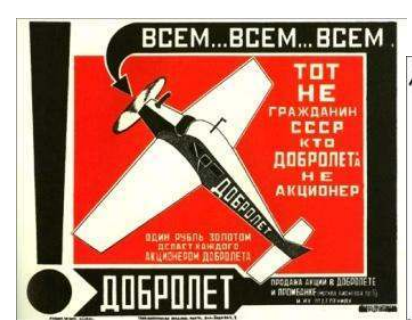

Rodtchentko 1924

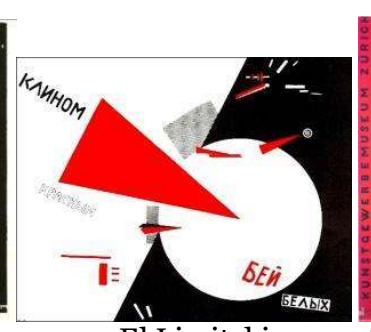

El Lissitzki 1920 et 1930

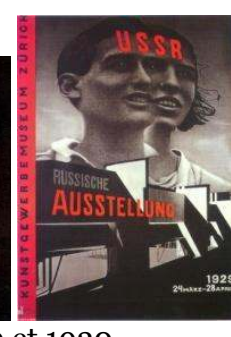

De même qu'on ne peut s'empêcher de penser au drapeau anarchiste ou à la svastika nazi, où là encore l'esthétique est liée à des fins propagandistes.

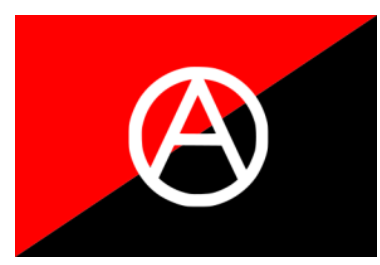

Ces utilisations politiques ont sans aucun doute favorisé l'association de ce code graphique à l'idée d'une détermination militante, que l'on retrouve jusqu'à aujourd'hui, parfois à titre de détournement ludique (Eg. No Logo, Naomi Klein), ou tout simplement pour la clarté de son impact sensoriel (Eg. Youtube, ou plus simplement la signalétique de codification routière). 


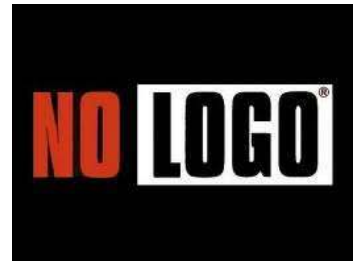

You Tube

Ainsi, on peut toutefois voir dans Mad Men d'autres formes de référence, comme par exemple l'évocation du graphisme du logo de Life, magazine phare de l'époque. On peut également l'interpréter comme un clin d'oeil intertextuel, une référence en miroir à une autre série contemporaine, débutée 3 ans avant Mad Men :

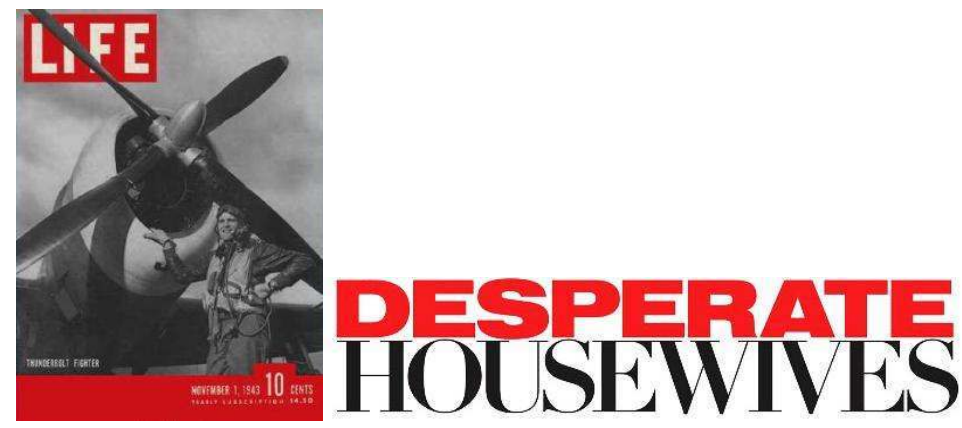

On notera le parallèle entre le titre de ces deux séries, jusque dans la phonologie ( 2 syllabes +2 syllabes vs. 1 syllabe+1 syllabe pour Mad Men), la syntaxe (Adjectif en rouge + Nom en noir), la sémantique (Appréciation négative + catégorie féminine vs. masculine).

Enfin, à titre de transition, revenons un instant sur le code couleur pour évoquer un mouvement esthétique ayant largement exploité la composition noir-blanc-rouge : le Bauhaus. 


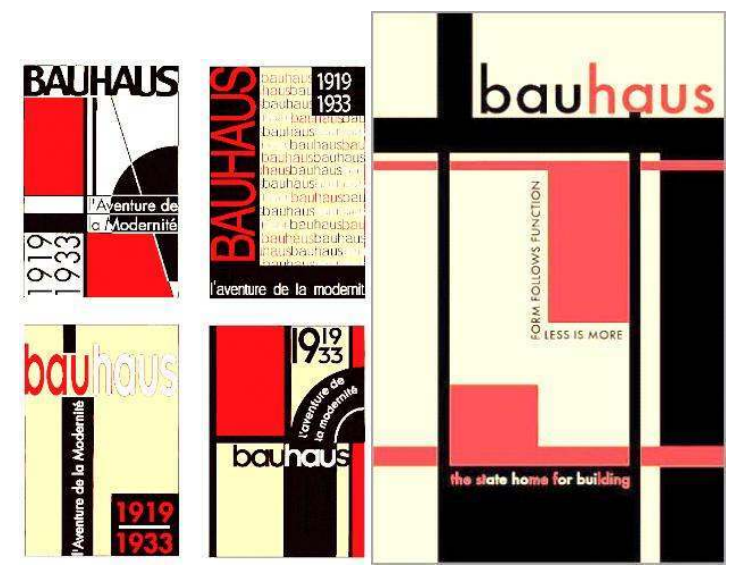

On se souvient qu'un des mots d'ordre du Bauhaus, et non des moindre, fut le célèbre : Less Is More, principe en réalité formulé au 19e siècle par Robert Browning mais remis au goût du jour dans les années 20 par un des chefs de file de l'école Bauhaus, Mies Van der Rohe. Or, on ne peut s'empêcher de voir une certaine ironie entre le code couleur de la logographie de Mad Men et l'association de ce même code à un mouvement incitant au minimalisme, car nous allons le voir plus loin, Sterling Cooper (l'agence de pub de Mad Men) est aux antipodes du minimalisme, qui fait pourtant son retour en 1960, justement dans le monde publicitaire.

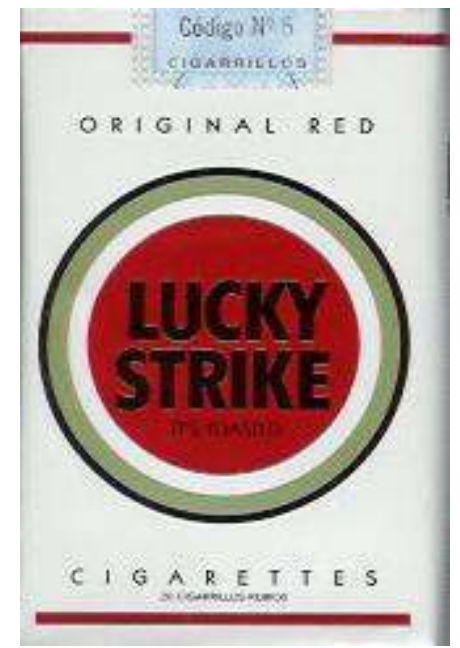


L'ironie se trouve par ailleurs renforcée lorsqu'on sait que le premier épisode de la série met en scène une campagne publicitaire pour Lucky Strike, dont le packaging du designer Raymond Loewy (1942) n'est pas sans rappeler lui aussi un certain code couleur.

C'est sur cette double ironie que je voudrais maintenant m'arrêter, afin de montrer dans quelle mesure l'agence publicitaire Sterling Cooper, théâtre même du microcosme autour duquel gravite toute la série, est posée d'emblée dans une relation d'altérité avec son époque.

\section{Sterling Cooper : l'autre agence.}

La série débute donc avec une campagne publicitaire pour Lucky Strike. C'est l'occasion de camper le principal protagoniste de la série, Don Draper, comme un directeur de création intuitif, inspiré et talentueux, du moins le plus inspiré au sein de Sterling Cooper. En effet, dans ce premier épisode les responsables de Lucky Strike viennent pour un état de l'avancement du projet alors que l'industrie du tabac doit maintenant faire face à une législation qui interdit de présenter le tabac comme un produit bénéfique pour la santé. Excédés par le fait que personne chez Sterling Cooper n'est en mesure de proposer ne serait-ce qu'un début de slogan, les annonceurs - Lee senior et Lee Junior - quittent la table dans un mouvement de colère. C'est alors que Don Draper est frappé par un éclair de lucidité, $a$ «lucky strike » pourrait-on dire:

DON DRAPER: Gentlemen, before you leave, can I just say something?

ROGER STERLING: I don't know, Don. Can you?

DON DRAPER: [To the clients] The Federal Trade Commission and Readers Digest have done you a favor. They've let you know that any ad that brings up the concept of cigarettes and health together...well, it's just going to make people think of cancer.

LEE [annonceur]: Yes, and we are grateful to them.

DON DRAPER: But what Lee Jr. said is right. You can't make those health claims. Neither can your competitors.

LEE [annonceur]: So...we got a lotta people not sayin' anything that sells cigarettes.

DON DRAPER: Not exactly. This is the greatest advertising opportunity since the invention of cereal. We have six identical companies making six identical products. We can say anything we want. [Pointing to Lee Jr.] How do you make your cigarettes?

[....]

LEE [annonceur]: We breed insect-repellant tobacco seeds. Plant 'em in the North Carolina sunshine. Grow it, cut it, cure it, toast it. . . 
DON DRAPER: There you go! [He writes « It's Toasted » on the blackboard.]

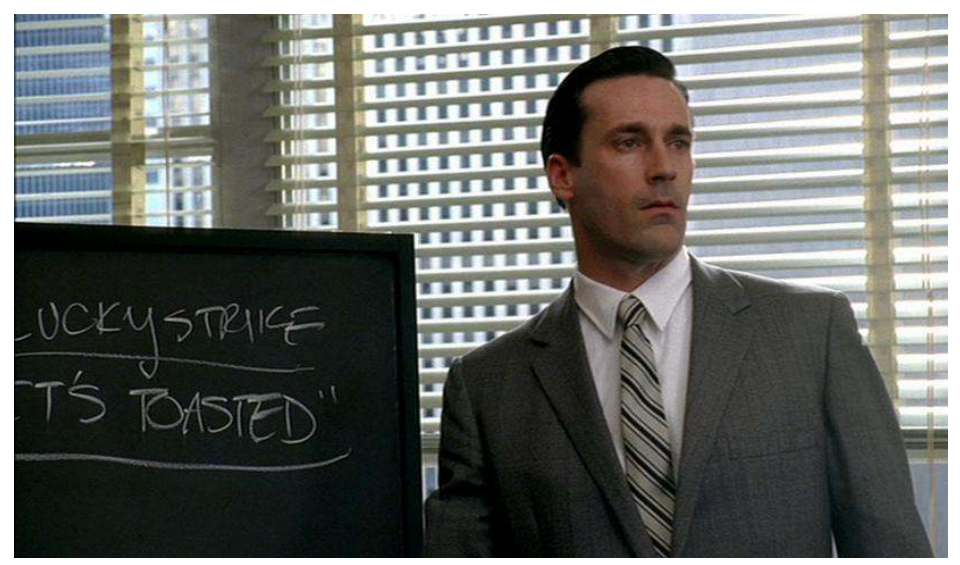

LEE JUNIOR [annonceur]: But everybody else's tobacco is toasted.

DON DRAPER: No. Everybody else's tobacco is poisonous. Lucky Strike's is toasted.

Contrairement aux apparences, cette scène ne nous présente pas tant Don Draper comme un publicitaire de talent que comme un personnage opportuniste, prompt à s'adapter à la situation et à en tirer profit. Il faut en effet savoir que It's Toasted est en fait un slogan associé à la marque dès $1917^{2}$ :

${ }^{2}$ http://en.wikipedia.org/wiki/Lucky_Strike 


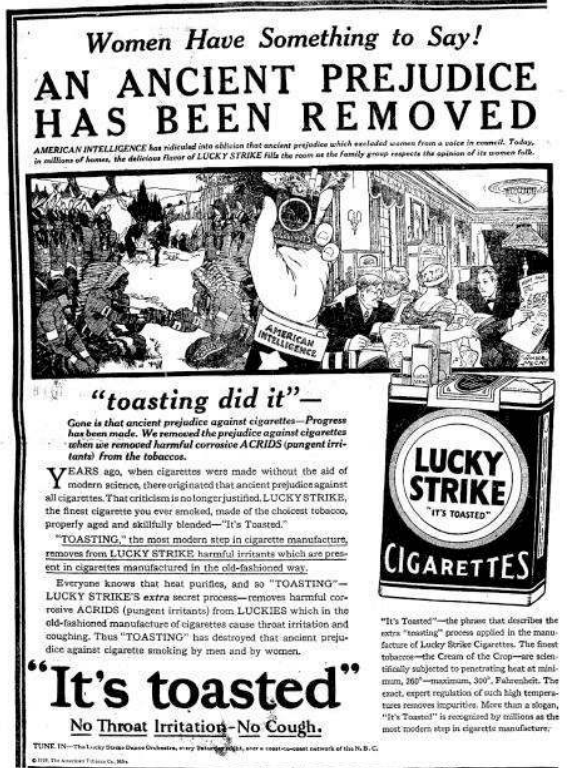

La mise en avant d'un argument de marque distinctif est en fait héritée des techniques de Claude Hopkins (1866-1932), revisitées par l'avocat du hard-sell Rosser Reeves dans les années 40 sous la forme de l'Argument Unique de Vente (Unique Selling Argument) :

This was the essence of the Hopkins approach. For each product, he would find the unique factor that set it apart from its rivals. (Tungate 2007:19)

Ainsi, pour reprendre la démonstration de Don Draper: " Everybody else's tobacco is poisonous. Lucky Strike's is toasted ».

On assiste par conséquent à la mise en application d'une technique créative datée, qui tendrait plutôt à présenter Sterling Cooper comme une agence traditionnelle, opérant selon des approches normées, académiques.

Or, rappelons-le, la scène se déroule en 1960. Année que l'on peut justement associer aux débuts de la " révolution créative » du monde publicitaire, période de laquelle émerge un nom par-dessus tous les autres, celui d'un autre publicitaire de Madison Avenue, celui d'un autre Mad Man : William Bernbach. 


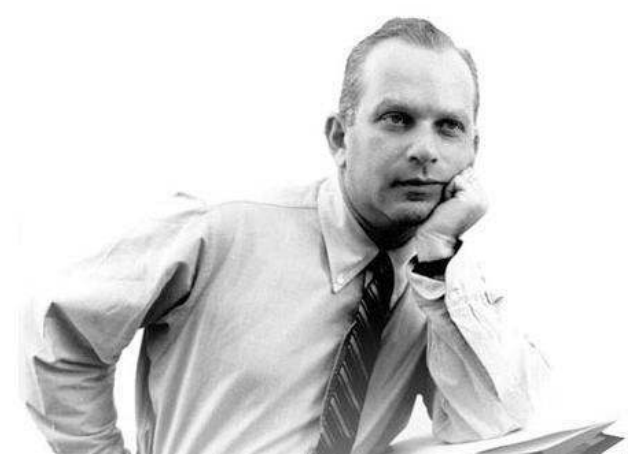

Doyle Dane Bernbach (DDB), l'agence crée dès 1949 par William Bernbach, est en quelque sorte un double en négatif de Sterling Cooper. Loin d'être une agence traditionnelle peuplée de WASP formatés par leur passage dans les grandes écoles, DDB a un directeur artistique juif (les remarques antisémites ne sont pas rares parmi les cadres de Sterling Cooper), qui prône un anticonformisme créatif et appréhende la publicité comme un art plutôt que comme la mise en application d'un technique :

Bernbach was a big noise in town because he'd set out to challenge the monolithic pre-war agencies that now dominated Madison Avenue. [...] Bernbach felt that their ads had become 'dishonest, boring, insulting even insane'. He argued that the repetitive tactics of people like Rosser Reeves had reduced the industry to one poor tired little ad' and the the giant agencies were 'turning their creative people into mimeograph machines'. Unless advertising shook up its ideas, he warned, it would become invisible, with zero impact on consumers. (Tungate 2007: 49)

Ou, pour citer Bernbach lui-même :

Advertising is fundamentally persuasion and persuasion happens to be not a science, but an art. (cité dans Levenson 1987)

Si Bernbach rencontre quelques premiers succès avec les magasins Orbach's ou encore la compagnie aérienne El Al, c'est avec sa campagne Volkswagen qu'il va définitivement graver son nom dans l'histoire de la publicité. Il faut pour cela comprendre que la Coccinelle est un produit a priori invendable sur le marché américain : elle est peu élégante, petite, sans fioriture, et de surcroît elle est associée à l'Allemagne Nazie. Or Bernbach, publicitaire juif, va contribuer à l'explosion des ventes de ce modèle grâce à une campagne qui va 
ébranler les habitudes du monde de la publicité. A cette époque, le marché de la voiture fonctionne sur le mode de la surenchère : toujours plus gros, toujours plus rutilant :

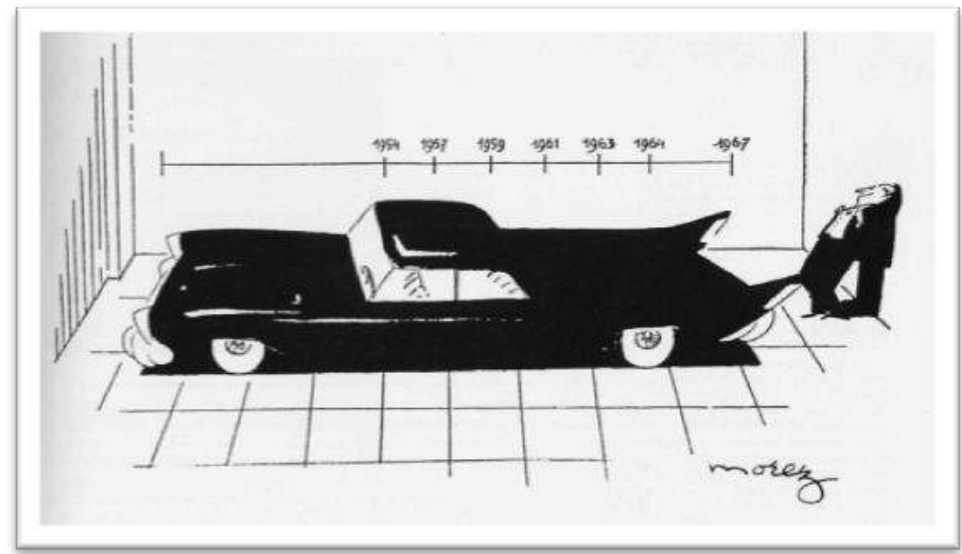

Tiré de Darmon et al., 1993.

Les publicités de l'époque sont hautes en couleurs, souvent dessinées, ce qui permet un meilleur contrôle de l'agencement des composants à une époque où la retouche photo n'est pas aussi flexible qu'aujourd'hui, associant généralement la voiture à la représentation d'une famille ou d'un voisinage idéalisé : on est dans l'ère du lifestyle advertisement

Pontiac's Beauty is Pontiac's Alone!
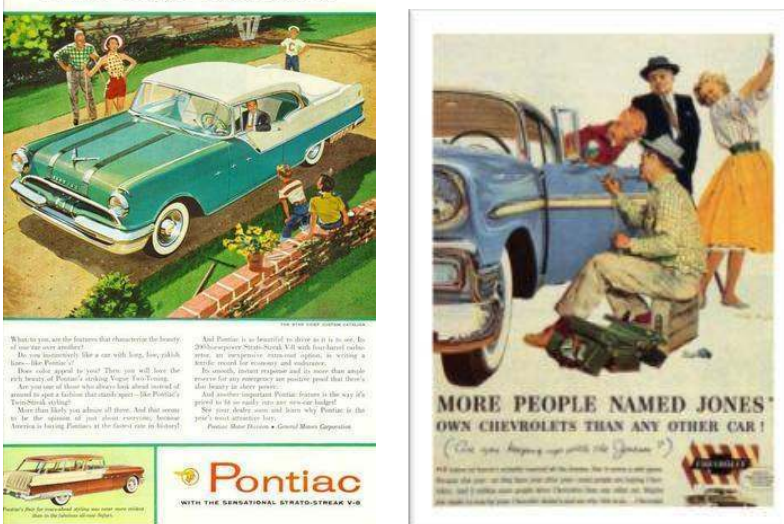

Tirés de Heimann, 2001. 
Le voisin, 'The Joneses', est le support privilégié d'un discours émulatoire dans une époque soucieuse de son appartenance et de ses valeurs sociales.

On peut dire que Bernbach va prendre le contrepied de tout. Aux grosses voitures, il substitue une miniaturisation, à la couleur le noir et blanc, au dessin la photo, au groupe social une désindividuation, au point d'exclamation un simple point, au lifestyle advertisement le mindstyle advertisement. Car si l'on ne peut ni changer le produit ni changer le marché, alors il faut changer les mentalités : Think small.
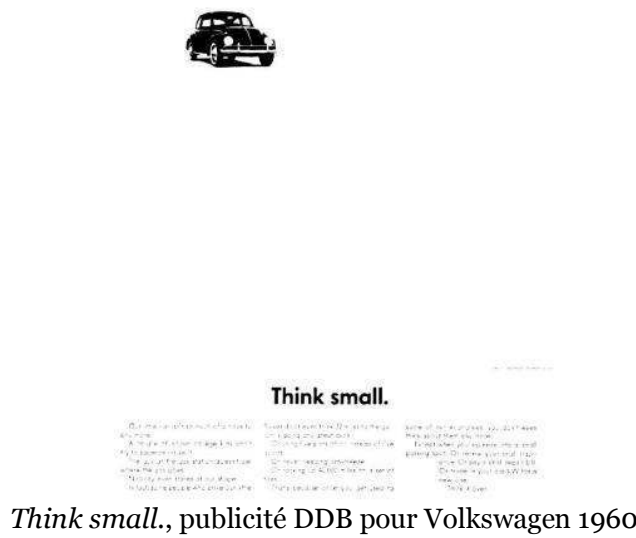

Du jamais vu : on est en 1960. On a épuré le message de toute mise en forme qui ne fait pas sens : Less is more. Tout doit avoir une fonction, jusqu'à ce point qui fait son apparition à la fin du slogan. Le point, le symbole le plus... petit. Quelque part sur Madison Avenue, une agence est déjà en train d'écrire les années 60, mais cette agence n'est pas Sterling Cooper. Voici au contraire le type de mise en page proposée par Don Draper dans l'épisode 4, affiches que même l'annonceur rejette tant il les trouve ringardes : 


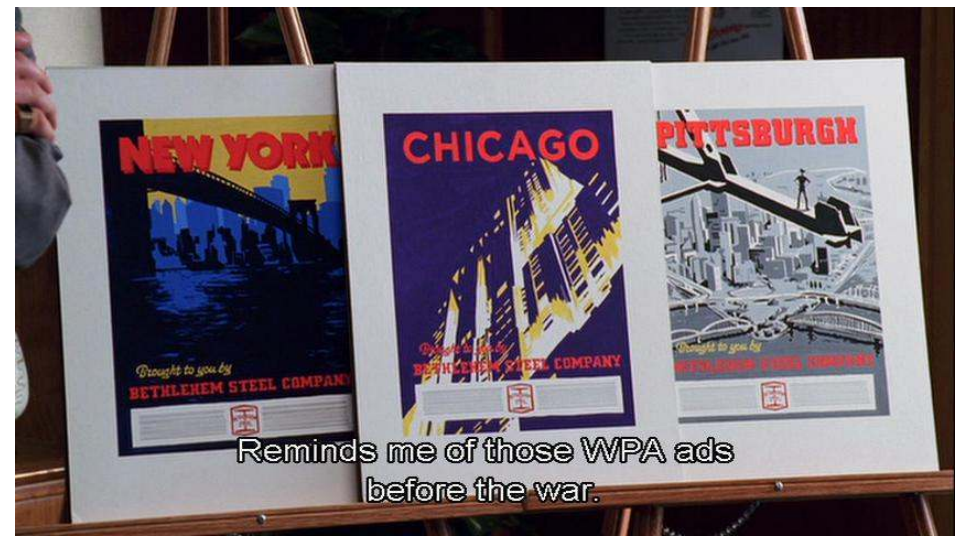

On est loin du retour minimaliste initié par Bernbach.

Bien que Sterling Cooper soit une agence fictive, créée pour les besoins de la série, Bernbach y apparaît en arrière-plan à plusieurs reprises, comme pour mieux mettre en évidence les défauts de Sterling Cooper. Ainsi, lorsque la représentante de $\mathrm{El} \mathrm{Al}$ vient dans les bureaux de Sterling Cooper, non seulement parle-t-elle de Bernbach, mais on ne peut s'empêcher d'y voir un clin d'œil à une publicité El Al de DDB 1957 (jusque dans le détail de la broche3) :
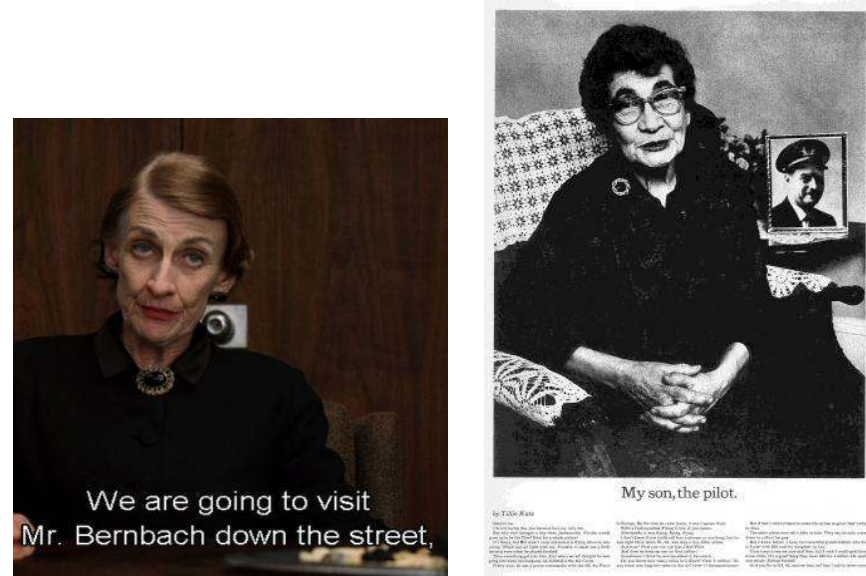

Episode 6

${ }^{3}$ Tiré de Darmon et al., 1993. 
Plus directement, le travail de Bernbach est directement commenté, avec un mélange d'admiration et de mépris, au sein de Sterling Cooper :

Roger STERLING: "Bernbach, he's a Jew... if I were him I wouldn't want to help re-industrialize Germany."

SALVATORE ROMANO: "Everybody's got a price."

PETE CAMPBELL: "Oh yeah, I saw that... honesty, it's a great angle."

SALVATORE ROMANO: "No chrome, no horsepower, horn... ugly, where's their strengths?"

PETE CAMPBELL: "It is funny!"

PAUL KINSEY: "It is?"

HARRY CRANE: "Cause I think the jokes on us; you're supposed to look at that and say it's a great car, not a great ad."

[...]

DON DRAPER: "Well, say what you want, love it or hate it, the

fact remains we've been talking about this ad for the last 15 minutes."

Il nous est montré que Don Draper semble apprécier le travail de Bernbach, ou du moins il lui reconnaît sa valeur pragmatique ; on le voit ainsi découvrir la pub Lemon 4 de 1960 dans le train, avant que n'ait lieu la discussion chez Sterling Cooper :

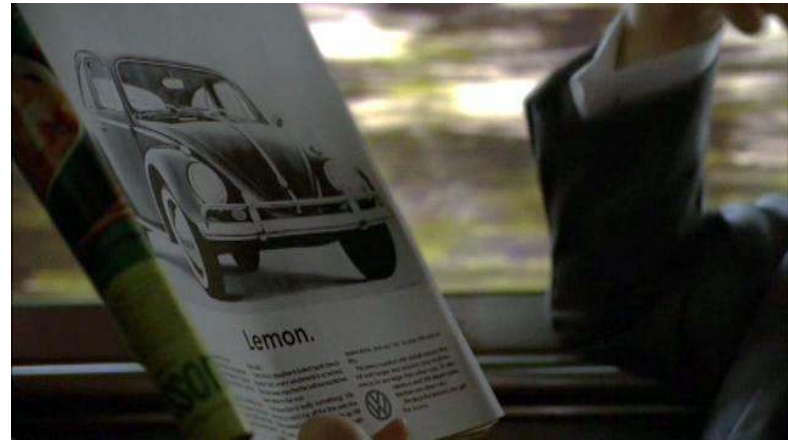

Episode 3

${ }^{4}$ Lemon est un jeu de mot qui fait à la fois référence à la forme de la voiture et à ce que l'on pourrait traduire par "pépin » (au sens de «problème », sens qui est clairement exploité dans la copy). 


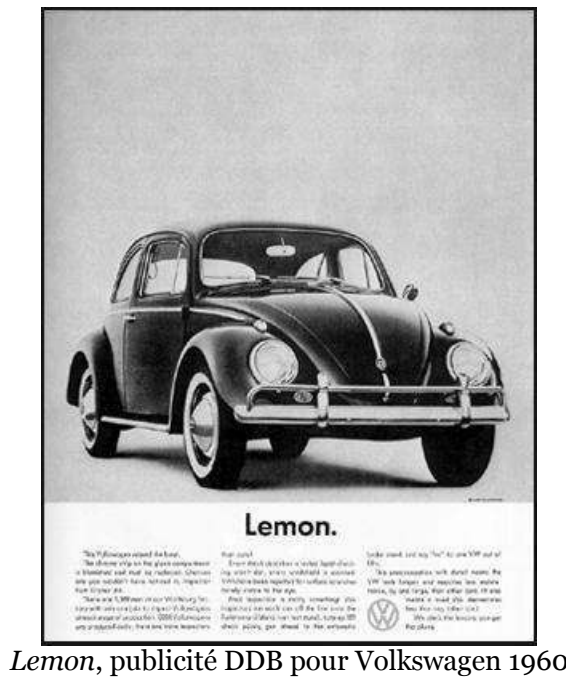

Don Draper est à l’image du publicitaire lambda de l'époque : admiratif peut-être, envieux sans doute, mais plus probablement encore frustré face à cette révolution créative qui dicte désormais les règles d'un jeu dont il se croyait jusque-là l'arbitre immuable.

Si Bill Bernbach peut être vu comme un double en négatif de Don Draper, il n'est pas le seul double auquel on puisse penser. En réalité, le protagoniste est défini non seulement intrinsèquement, par la manière dont il agit, mais aussi extrinsèquement, en vertu de sa relation avec tout un réseau de personnages qui le définissent de l'extérieur. C'est sur ce point que nous nous arrêterons dans la partie qui suit.

\section{Don Draper, publicitaire entre autres.}

On peut tenter d'organiser les différents doubles de Draper selon une sémiotique de dichotomies. D'une part, on peut distinguer des doubles intra-fictionnels comme Dick Whitman, Adam Whitman et Pete Campbell (tous créés pour la série), et des doubles extrafictionnels comme Bill Bernbach ou Draper Daniels, sur lequel nous allons revenir. Par ailleurs, on peut reconnaître d'un côté des doubles positifs et des doubles négatifs (on vient de voir en quoi Bernbach pouvait être considéré comme un double négatif). Enfin, on peut également distinguer les personnages qui vont définir Donald Draper dans sa sphère privée et ceux qui le définissent dans sa sphère 
professionnelle, ces deux terrains étant relativement compartimentés dans la série 5 :

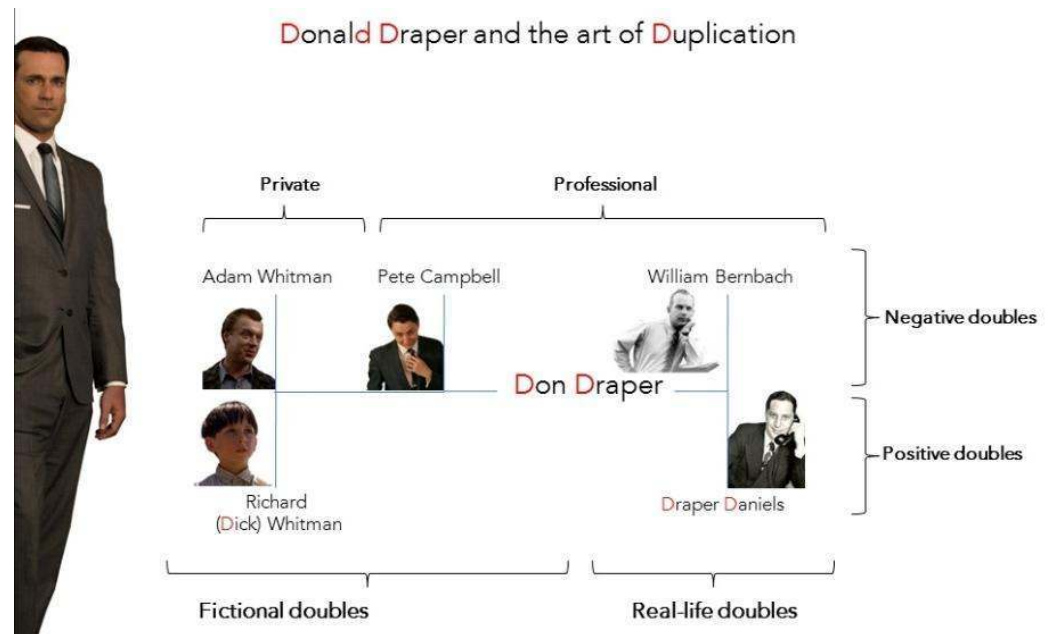

- Donald Draper et Draper Daniels
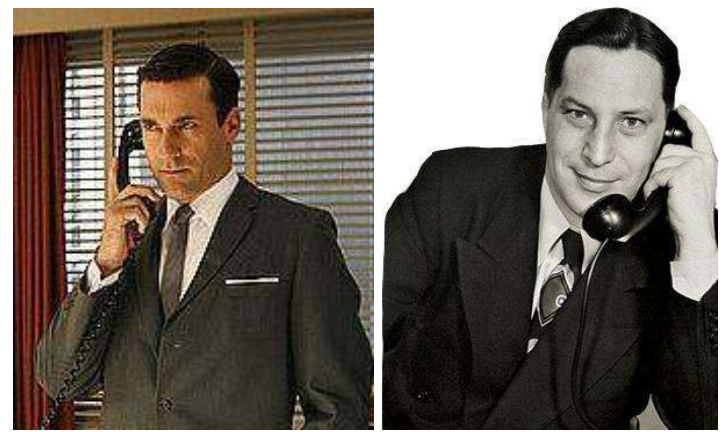

Les similitudes entre Draper Daniels et Donald Draper, entre Dan et Don, sont nombreuses, et dépassent de loin leurs patronymes ou leurs traits physiques. De ce point de vue, Matthew Weiner est resté assez fidèle à son modèle en élaborant son personnage. Comme Don, Dan fumait beaucoup, buvait beaucoup, courtisait beaucoup. Et pour

5 Tableau tiré du Powerpoint de ma communication orale du 8 juin 2010. 
ce qui est des détails moins immédiats, Draper Daniels venait d'un milieu fermier et travaillait comme directeur de création pour Leo Burnett. Aussi, est-il important de dire un mot de l'agence Leo Burnett. A la différence des grandes agences de l'époque, Leo Burnett n'était pas localisé sur Madison Avenue, mais à Chicago, et de fait sa publicité a toujours été en marge, avec un style détaché du microcosme mondain de New York.

Now I don't intend to argue that Chicago is in any way a worthier city than, say, New York. But I am suggesting that our sod-busting delivery, our loose-limbed stand and our wide-eyed perspective make it easier for us to create ads that talk turkey to the majority of Americans - that's all. (Leo Burnett)

Leo Burnett a notamment une technique particulière pour donner au produit un mode d'appréhension plus intuitif, plus immédiat : lui associer un représentant graphique. On lui doit ainsi le Géant Vert (1925), Tony le tigre (1951) ainsi que tout le bestiaire des céréales Kellogg's, ou encore le cowboy Marlboro (1954). Ce dernier est en réalité l'œuvre de Draper Daniels. On voit ainsi se dessiner, à travers ce modèle extra-fictionnel, un personnage doté d'une double personnalité : à la fois urbain et raffiné mais obéissant par ailleurs à des intuitions et à une pragmatique liées notamment à des origines que, contrairement à Draper Daniels, Donald Draper renie totalement.

\section{- Donald Draper et Dick Whitman}

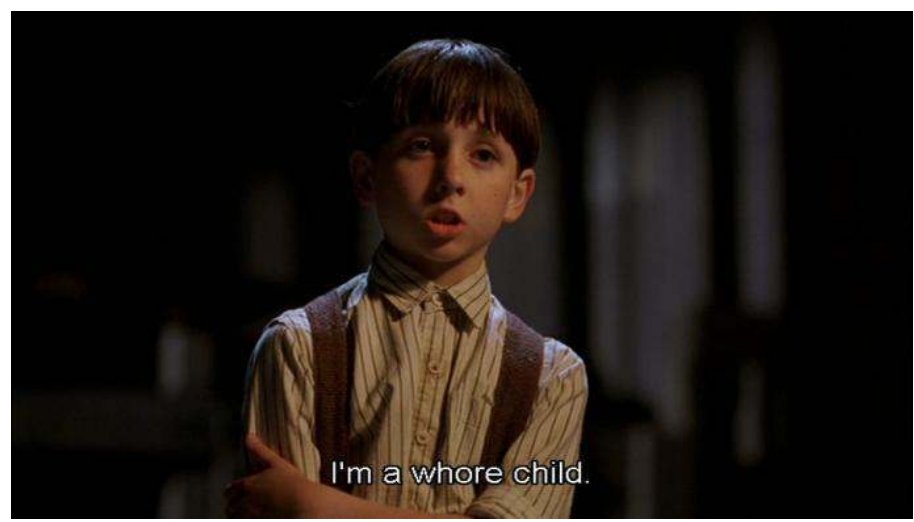

Episode 8 : Dick Whitman - Donald Draper enfant -

se confiant à un étranger de passage.

Les retours sur l'enfance occultée de Don Draper posent le problème de l'identité en diachronie. Si l'on peut parler de double 
positif, c'est parce qu'il a une continuité existentielle entre la personne de Dick Whitman et celle de Don Draper. Cela étant dit, la dissymétrie de l'orientation chronologique induit de facto une altérité : Dick n'est pas Don, il en est une version en construction, un épisode préparatoire. Pour autant, les indications apportées par ces flashbacks participent pleinement de la construction d'un personnage, dont les origines sont en rupture avec sa situation d'arrivée. Dick est en quelque sorte le point de fuite qui replace la vie de Don Draper dans toute sa perspective. From Dick to Don; from duck to swan.

\section{- Donald Draper et Adam Whitman}

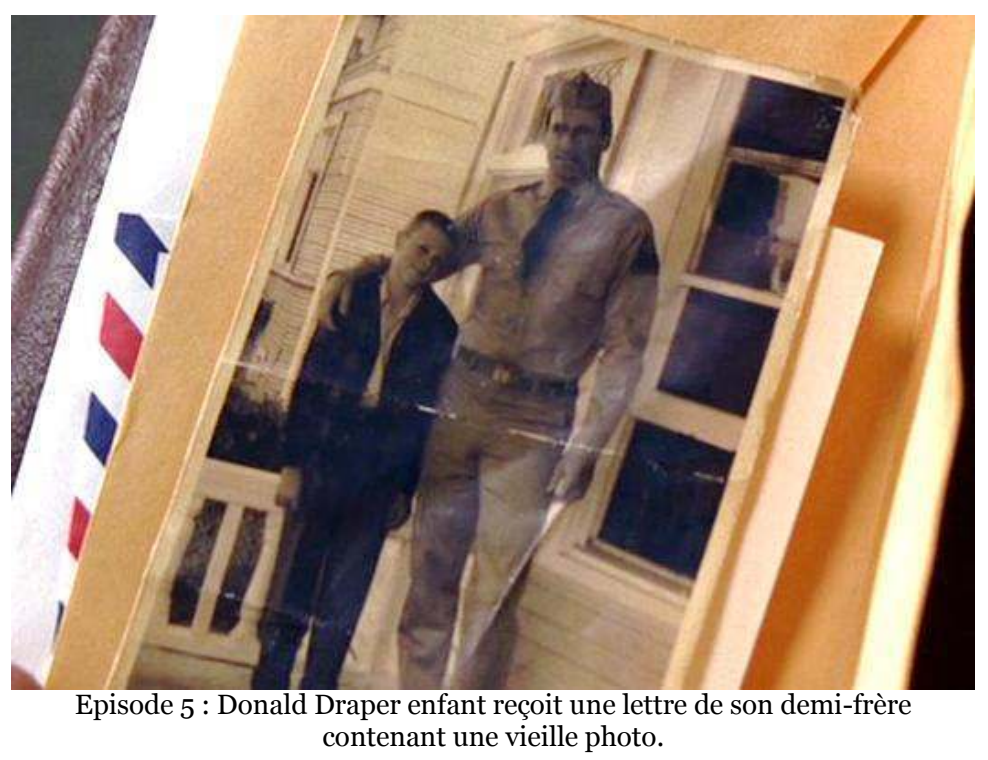

Adam Whitman, le demi-frère cadet de Don Draper, est le seul personnage de la série qui connaisse le passé de Don Draper. En soi, Adam Whitman n'est pas vraiment un double de Don Draper, mais il est le catalyseur qui va nous faire accéder à sa facette la moins noble. Rejetant son propre frère dont il tente d'acheter le départ, Don Draper va précipiter celui-ci vers le suicide. Si le personnage de Dick Whitman recréait dans la chronologie un point de fuite, Adam Whitman s'apparente plus à un point de chute, à un abîme affectif qui confère au protagoniste ses dimensions les plus insondables.

Qu'il s'agisse d'Adam ou de Dick, cette ouverture vers un passé enfoui et inavouable fait émerger une des représentations les plus saisissantes de l'altérité dans la série. Elle est une dramatisation de ce 
conflit universel qui place chacun de nous face à son autre soi. De ce passé qui n’a pas sa place dans le présent, mais qui n'a de cesse de le revisiter et de rappeler... where the truth lies.

\section{- Donald Draper et Pete Campbell}

Si Donald Draper est le prototype du self-made man, Pete Campbell en est l'exact contraire : issu de la bourgeoisie WASP, produit des grandes écoles, les deux personnages apparaissent sous des relations conflictuelles à de nombreuses reprises (ici épisode 12):

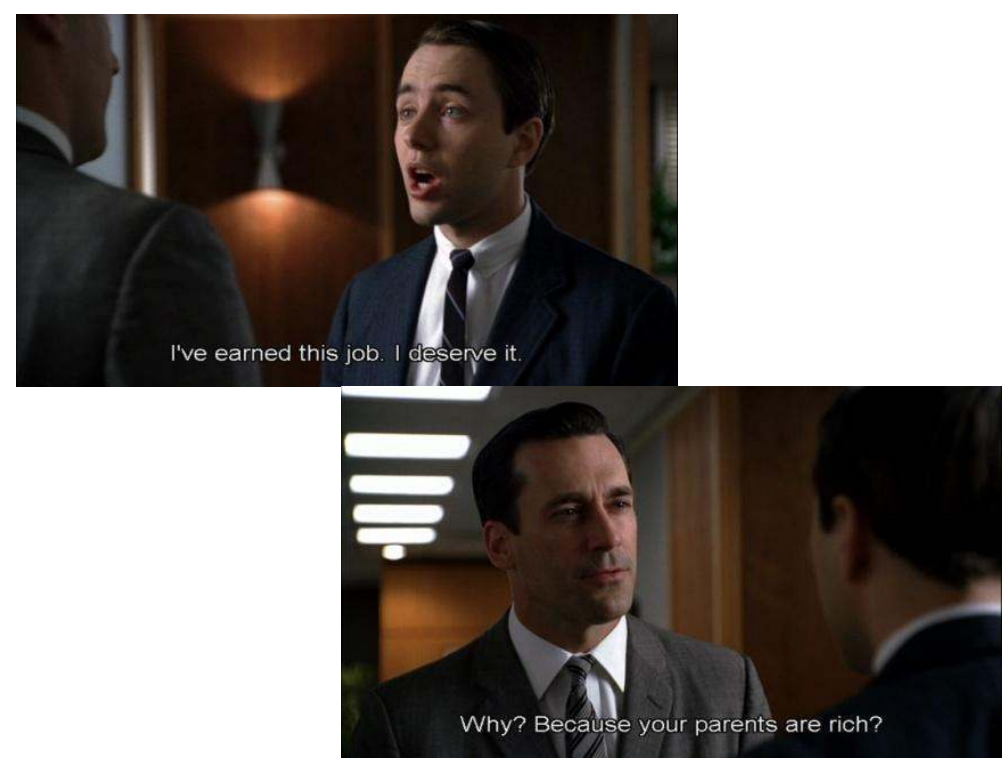

Les différences entre les deux personnages sont tout au profit de Don Draper, qui se trouve valorisé dans la mesure où toutes les facettes négatives de Campbell font écho chez Draper à une contrepartie positive, par contraste. De manière intéressante, même dans ses aspects les plus critiquables, Don Draper ressort en quelque sorte amendé du fait de la comparaison avec Campbell. Par exemple, on sait que l'épisode de la Corée (épisode 12) est peu glorieux pour Draper puisque c'est à ce moment qu'il usurpe l'identité de son supérieur, tué au combat suite à une négligence de Draper lui-même : 


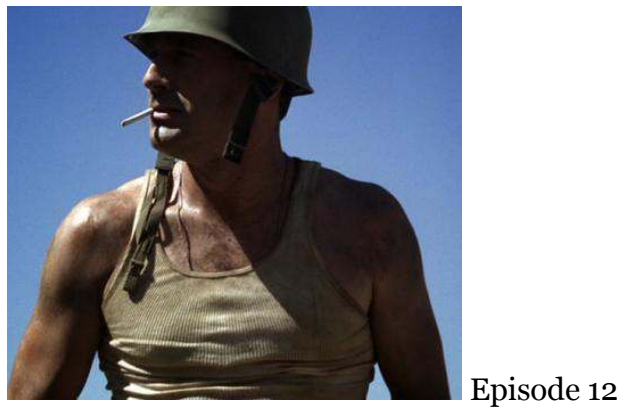

Pour autant, l'expérience militaire de Draper nous apparaît comme une facette authentique et constitutive de sa personnalité ; expérience que Campbell ne peut que contrefaire, se présentant une fois de plus comme un personnage vain et superficiel, comme lorsqu'il exhibe sa carabine dans l'agence :

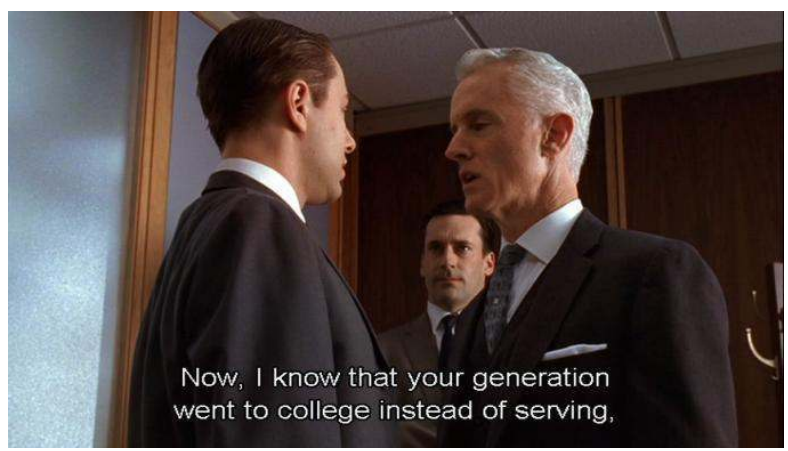

Episode 4

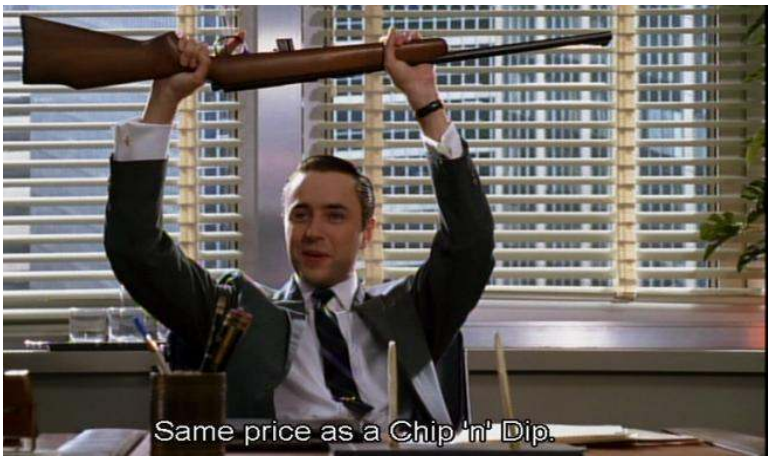

Episode 7 
De fait, la seule utilisation que Campbell semble apte à faire de ce " cadeau de mariage » - qu'il a choisi après avoir retourné un très kitsch service à dips - est de viser des proies féminines (pour ce qui est d'une illustration de la notion de substitut phallique, nous voilà servis).

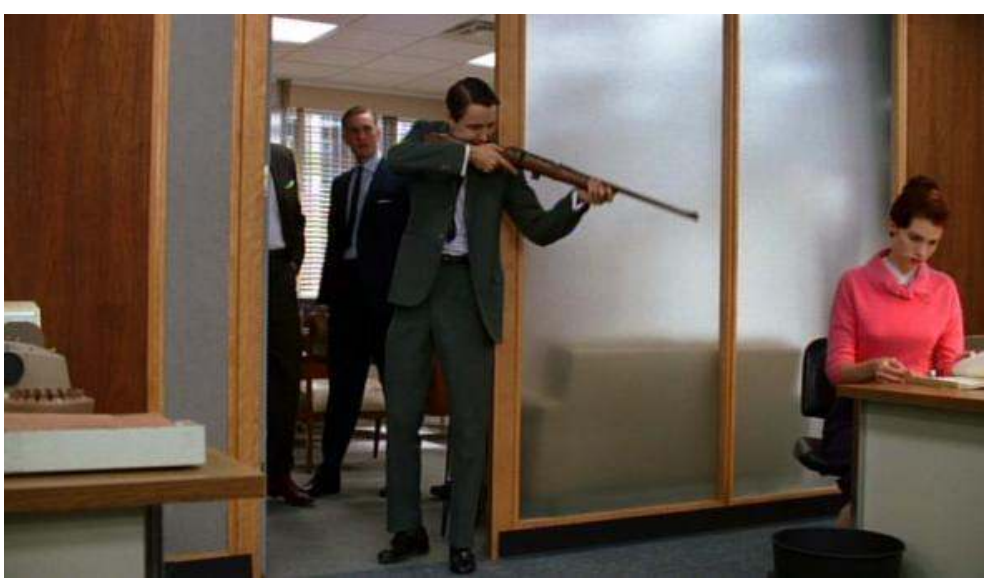

Et de fait, si le rapport de Don Draper vis-à-vis des femmes invite à la critique sur de nombreux points, il ressort là encore grandi par contraste avec le comportement de Campbell, qui manque de noblesse en tout point. De même, si Draper peut être vu comme un ambitieux qui est allé jusqu'à renier ses origines pour atteindre son objectif, on en vient finalement à considérer que cette ambition s'apparente à un souci d'élévation, alors que celle de Campbell s'apparente à un arrivisme pétri d'arrogance. Tout se passe comme si Campbell avait pour fonction de relativiser les dimensions les moins éclatantes de Draper en offrant une représentation pervertie de ces mêmes défauts.

\section{Mad Men's desperate housewives.}

Mad Men is about the conflicting desires in the American male, and the people who paid the price for that, who were the women. Matthew Weiner 6

La relation hommes/femmes est une composante fondamentale de Mad Men, et il faudrait au strict minimum consacrer un article à part entière à cette dimension. Les commentaires qui suivent sont par

${ }^{6}$ Matthew Weiner est l'écrivain et le producteur de la série. Ce commentaire tiré des bonus de la saison 1 sur le DVD 3 de la version américaine. 
conséquent tout à fait allusifs et ne laisseront qu'entrevoir la complexité de la question. Il s'agit essentiellement de rappeler que cette relation problématique occupe une place centrale dans la thématique plus générale de l'altérité.

Le titre même de la série laisse présager que ce sont les hommes qui vont investir le devant de la scène. Et de fait, aucune femme n'occupe une position véritablement dominante. L'altérité au sein du rapport hommes/femmes semble prendre deux aspects dans la série. D'un côté une relation de subordination, de l'autre une rupture, une déconnexion entre femmes et hommes, comme deux univers hermétiques.

La première forme de déséquilibre est pleinement illustrée par la place des femmes au sein de l'agence. Les hommes occupent des postes créatifs, dans des bureaux individualisés ; les femmes des postes administratifs dans des espaces ouverts. Si cette ligne est franchie, si une femme prend part à une fonction créative, par définition ça n'est plus - ça ne peut plus être - une femme à part entière. Ce scénario est illustré lorsque Peggy Olson fait la démonstration de ses talents de copywriter, une des tâches créatives suprême de la publicité de cette époque où le format guillotine standard réserve environ 2/5 de l'espace au texte, l'image occupant générale les 3/5 au-dessus de la copy.

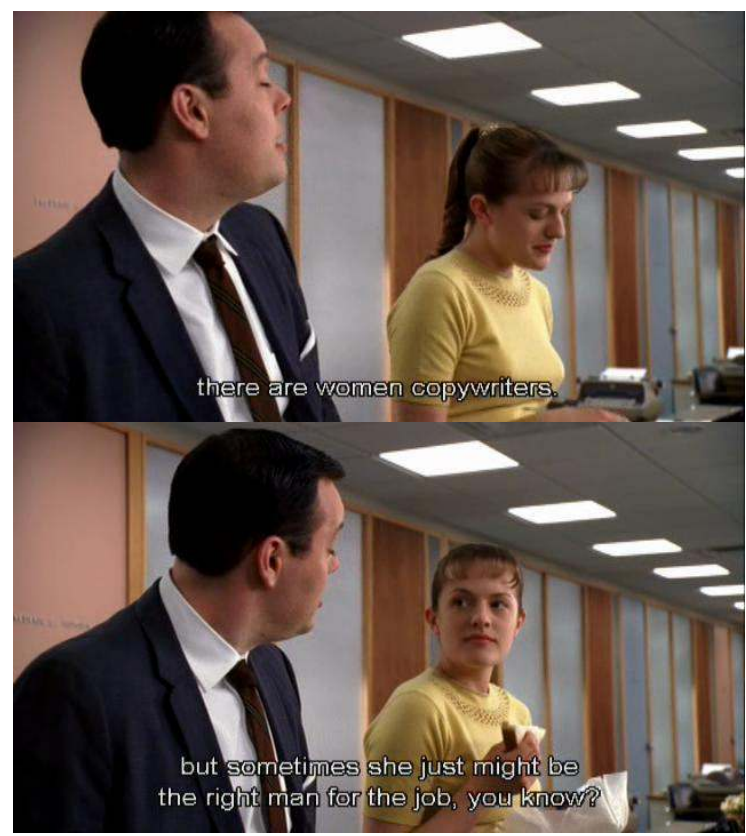




\section{Episode 2}

La recatégorisation de l'identité sexuelle de Peggy Olson est en quelque sorte une condition nécessaire à la préservation du modèle de société hiérarchisée, tel qu'il est imposé par la version conservatrice des années 50 : on bouge les pions, on ne change pas les règles. Il ne s'agit pas tant de voir en Peggy des qualités généralement associées aux hommes, que de reconnaître un homme en Peggy.

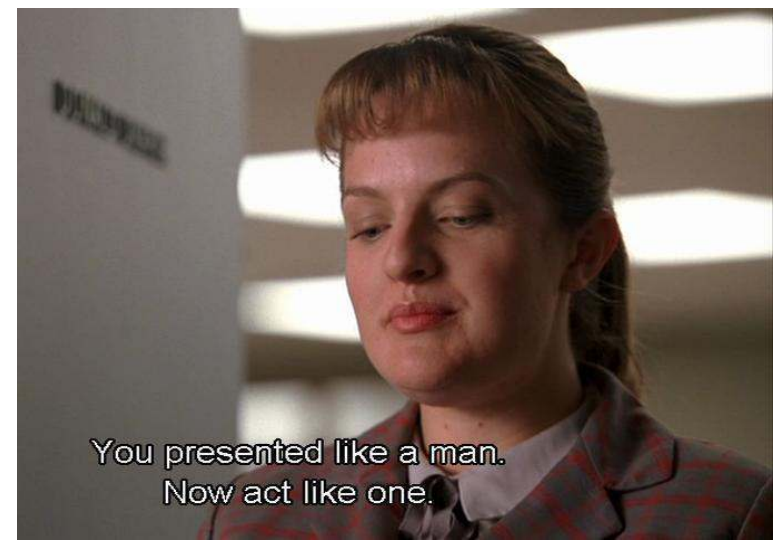

Episode 11

L'identité sexuelle et l'identité socio-professionnelle sont confondues au point que le déplacement de l'un doit s'accompagner du déplacement de l'autre. On ne peut passer de l'autre côté du miroir sans inverser son image. 


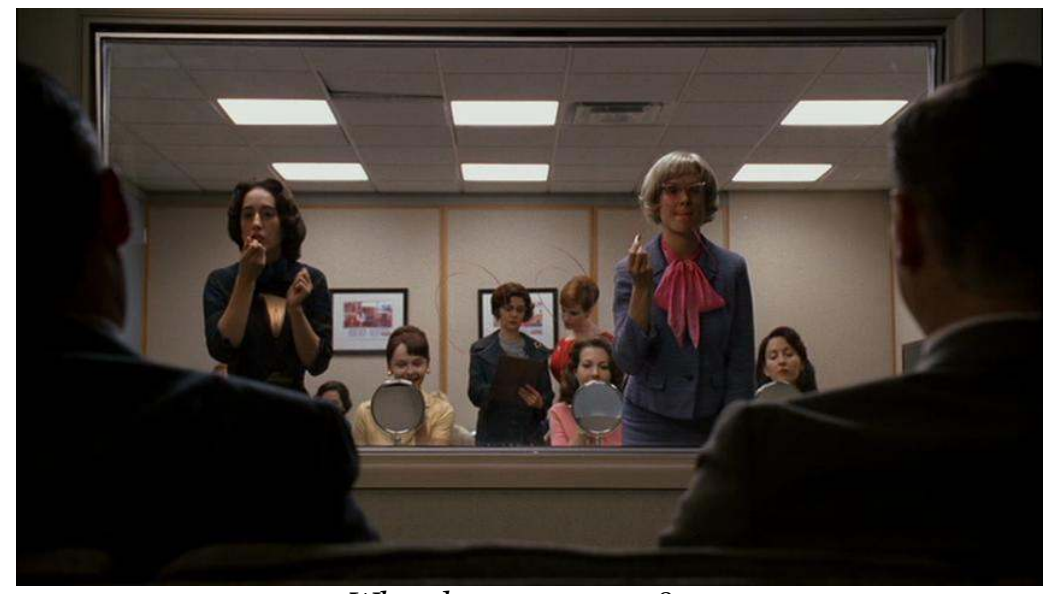

"What do women want?"

Episode 6: Les secrétaires de Sterling Cooper testent des rouges à lèvres pendant que les hommes étudient leur comportement derrière un miroir sans tain.

Comme je le mentionnais plus haut, il existe sans doute une seconde forme d'altérité dans le rapport hommes/femmes: la déconnexion entre ces deux communautés. La scène du miroir sans tain de l'épisode 6 est sans doute l'image la plus parlante de ce divorce culturel entre hommes et femmes. Deux mondes partageant un même lieu dans une incompréhension parfois totale, et qui rend les « hommes fous », notamment lorsqu'il s'agit de vendre des produits pour la clientèle féminine. Peggy Olson est, de ce point de vue, une sorte de cheval de Troie. Elle est la porte vers un univers dont les hommes ne perçoivent que la facette extérieure. Toujours à propos de la scène du miroir, on constate que, paradoxalement, le seul retour que les hommes reçoivent est en fait l'image de leur propre impuissance et de leur inintelligence face à celles qu'ils se donnent le droit de regarder impunément ; de les regarder, mais sans les voir.

Un autre mode de renvoi en miroir peut être commenté autour de Don Draper, dont la définition passe par sa relation aux femmes, et les différences de statuts que celles-ci entretiennent entre elles. Là encore on peut tenter de clarifier l'approche de ces personnages en organisant un carré sémiotique (je ne retiens que quatre personnages qui me paraissent centraux parce que en relation direct avec Don Draper) $:^{7}$

7 Tableau tiré du Powerpoint de ma communication orale du 8 juin 2010. 

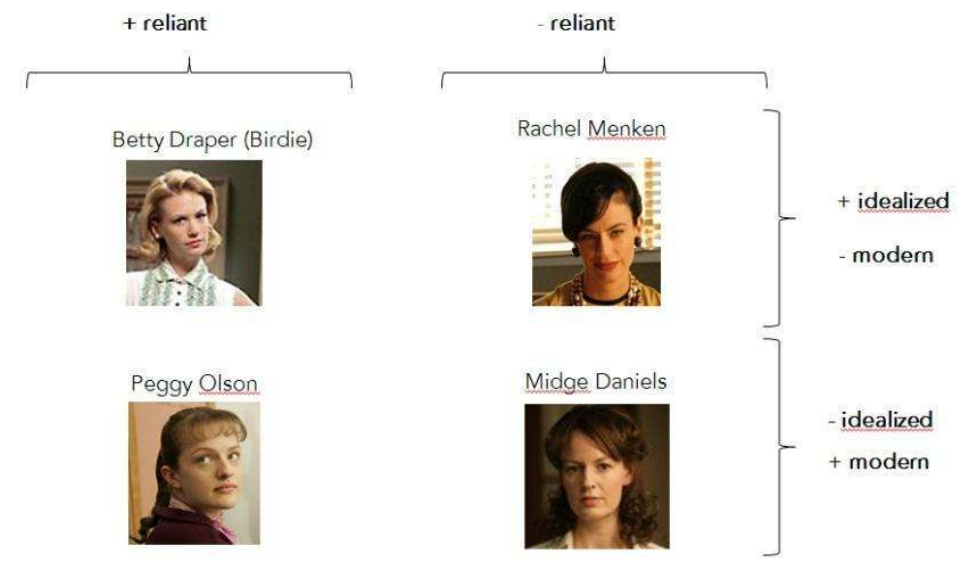

On peut distinguer d'une part le degré d'autonomie des personnages vis-à-vis de Draper. Ainsi, Betty Draper, en tant qu'épouse, dépend de Don Draper, ne serait-ce que financièrement, de même que Peggy Olson, en tant que secrétaire de Draper, est sous sa dépendance professionnelle. A l'inverse, Rachel Menken, héritière et directrice du grand magasin Menken's (on ne peut s'empêcher de penser à Orbach's le premier client de Bernbach), n'est pas sous la dépendance directe de Draper; le cas de Midge Daniels, une autre maîtresse de Draper, est plus discutable car elle reçoit ponctuellement de l'argent de Draper, mais elle est dans une relation assez libre avec Draper, au point qu'elle vit une existence plus ou moins parallèle au sein d'une communauté beatnik.

D'autre part, on peut distinguer le degré d'idéalisation des personnages féminins. D’un côté, Betty Draper et Rachel Menken, représentations standard de la femme des années 50, telle qu'elle pourrait apparaître dans une publicité de cette époque. D'un autre côté, Peggy Olson et Midge Daniels, qui préfigurent la femme des années 60 en route vers son émancipation professionnelle et intellectuelle, en décalage par rapport à ce modèle fantasmé dont Betty Draper est l'archétype.

\section{- Midge Daniels, Myra Djanco Daniels... et Peggy Olson}

De la même manière que l'on avait pu commenter le parallèle évident entre Don Draper et Draper Daniels, on ne peut s'empêcher de voir un rapport phonétique entre les patronymes de Midge Daniels et Myra Djanco Daniels, personnage extra-fictionnel, épouse du publicitaire Draper Daniels. Et pourtant, à y regarder de plus près, Myra Djanco Daniels est plus comparable dans son personnage à Peggy 
Olson qu’à Midge Daniels : Draper Daniels et Myra étaient collègues comme Don et Peggy, de même que Draper Daniels avait 12 ans de plus que Myra, Draper Daniels avait été marié avant de l'épouser, et Myra était devenue la première femme à diriger une agence de publicité au niveau national. Au point qu'on est en droit de se demander dans quelle mesure les deux dernières données ne trahiraient pas déjà la suite de la série.

\section{- $\quad$ Midge Daniels et Betty Draper}

Comme le laisse apparaître le carré sémiotique proposé plus haut, ces deux personnages sont diamétralement opposés. La brune et la blonde. La maîtresse et l'épouse. L'artiste indépendante du centre urbain et la mère au foyer de la banlieue bourgeoise. L'une passe des soirées à fumer de l'herbe avec ses amis beatniks, l'autre préfère les après-midis thé avec ses voisines. Le clivage est total entre la femme préfigurant les années 60 et celle incarnant les années 50. Sur le plan formel on trouve dans l'épisode 2 une belle illustration du rapport inversé de Draper vis-à-vis de ces deux personnages :

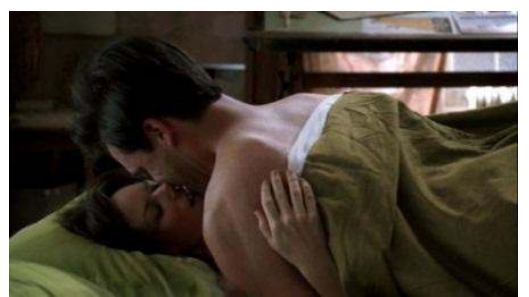

Don Draper couché faisant l'amour dans le lit de Midge Daniels ת

Don Draper couché faisant des pompes à côté du lit de Betty Draper

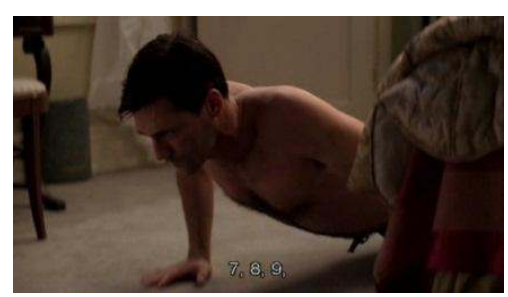

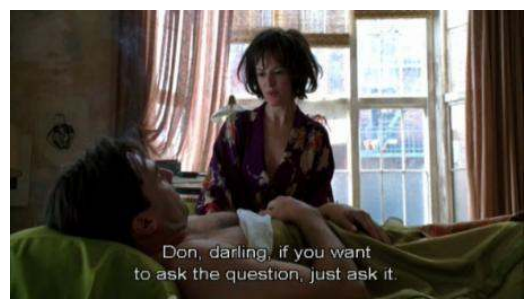

Midge Daniels debout, s'adressant par hypocoristiques à Don Draper allongé dans le lit ת

Don Draper debout, s'adressant par hypocoristiques à Betty Draper allongée dans le lit

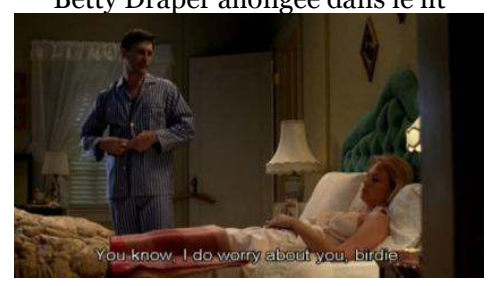

Encore une fois Don Draper se trouve défini extrinsèquement, en regard de son rapport à sa relation antagoniste avec ces doubles féminins : entre d'un côté une femme trophée imposée par son surmoi 
social et de l'autre une maîtresse rebelle en accord avec une attirance vers un univers plus subversif, qu'il explore tout le long de la série dans une double relation d'attraction et de répulsion.

Conflit qui habite également Betty Draper. A la différence que celle-ci enfermée dans sa cage dorée, n'a pas la latitude d'évasion de son mari. Une scène intéressante apparaît dans l'épisode 9 où Betty Draper fait une séance photo pour une publicité pour Coca-Cola :

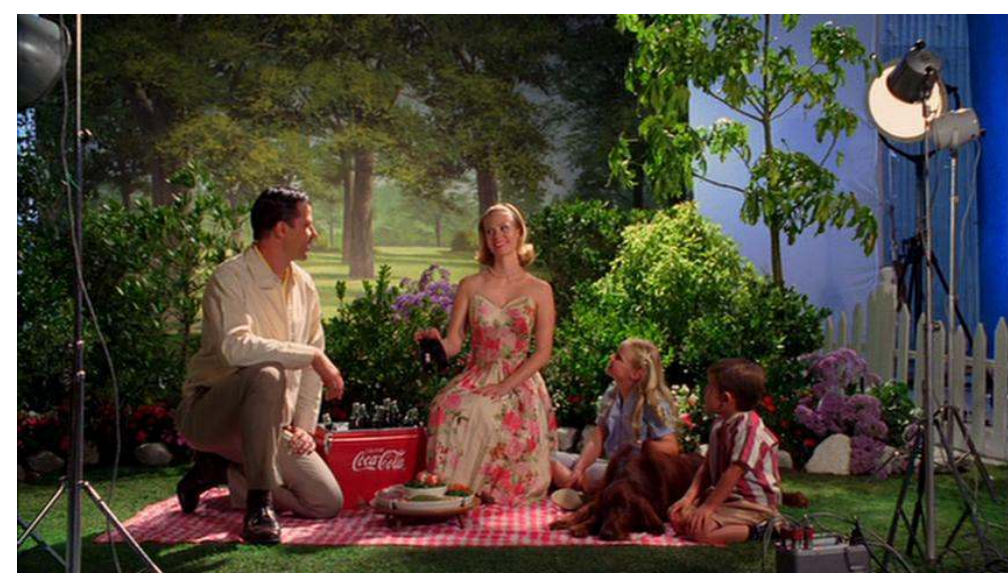

Betty Draper est tout en accord avec une mise en scène de la famille idéale des années 50. Plus qu'une femme des années 50, Betty Draper en est une caractérisation. Le figement du personnage dans cette champêtre de studio où la nature copie l'art résume à lui seul sa condition. A la fin du même épisode toutefois, Betty Draper, réalisant que la publicité ne serait pas diffusée en raison d'une querelle d'intérêt entre son mari et l'agence publicitaire qui a pris les photos, perd les pédales, sort avec une carabine à plomb, en nuisette, cigarette aux lèvres, et tire sur les colombes de son voisin : 


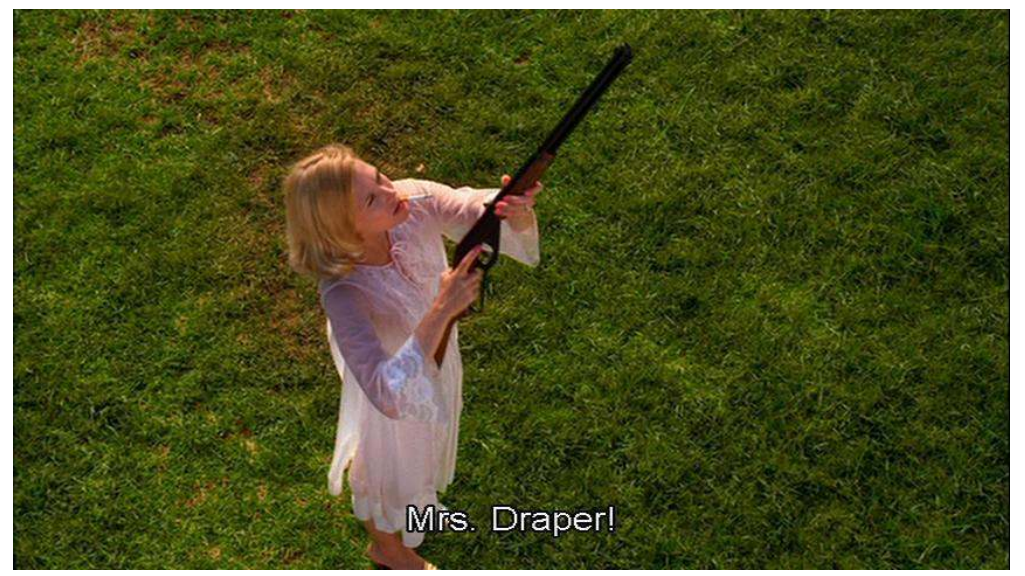

L'image de cette fin d'épisode est d'autant plus symbolique lorsque sait que son mari la surnomme Birdie. Birdie being shot. Birdie shooting. Ce geste de rébellion, fortement cathartique, sonne néanmoins comme un cri de désespoir plus que comme un acte de libération. Betty Draper est prisonnière de sa condition, de même que son mari est prisonnier de son personnage social. À chacun sa cage.

\section{The place where we ache to go again.}

What really makes Mad Men so special is that it presents a world that we kind of all yearn to go back to, but it's not an idealized world of nostalgia. Matt Roush ${ }^{8}$

Mad Men, on le comprend, est une vision dé-sublimée des années 50, dont on montre les coulisses, les arrière-cours ; un monde où les histoires d'amour ont l'aspect du vernis qui craque, où les héros sont prosaïques et imparfaits. Pour cette raison, il est intéressant que Mad Men se déroule dans le milieu de la publicité, à l'image du catalogue Taschen All-American Ads des années 50, qui répertorie des centaines de publicités pour des voitures, des parfums, des voyages, des frigos... Imaginons un instant que cet ouvrage soit un jour tout ce qui subsiste de notre civilisation. Que penserait-on de nous? Quelle image renverraient ces affiches où les gens sourient, où les hommes sont élégants et les femmes minces, où les jardins sont entretenus et les maisons rangées, où les rues ressemblent à des projets architecturaux, sans pauvres, sans noirs - du moins pas plus que dans les films

${ }^{8}$ Matt Roush est un critique de magazine télé. Ce commentaire tiré des bonus de la saison 1 sur le DVD 3 de la version américaine. 
d'Hitchcock ou de Billy Wilder de cette époque. Par essence, la publicité offre une représentation du monde, non tel qu'il est, mais tel qu'on souhaiterait qu'il soit. L'art du «faire vouloir » doit bien faire quelques arrangements avec le vrai. Hitchcock, puisqu'on vient d'en parler, disait qu'une bonne histoire, c'est la vie, dont on aurait retiré les parties ennuyeuses 9 . La publicité allait sans doute encore plus loin en ne retenant et en n'accentuant que les aspects positifs que nos vies. En nous emmenant visiter l'envers du décor, la série nous positionne à une interface : d'un côté, une forme de représentation magnifiée des années 50 que nous avons en mémoire, de l'autre côté une représentation de la vie ordinaire où ressurgissent les imperfections filtrées par le tamis de la pratique publicitaire. Ainsi, c'est la question du réel, de la représentation et du souvenir qui se pose. De ce point de vue la scène du Carrousel dans l'épisode final est particulièrement intéressante parce qu'elle ne fait pas appel à des images publicitaires, mais à des photos de la sphère privée qui représentent la saisie de moments heureux du passé de Don Draper. Cette scène est évidemment intense parce qu'elle invite par empathie chaque spectateur à se retourner vers une époque éphémère qui a été le théâtre de moments heureux. Or, on se rend compte que ces photos de famille sont elles aussi le produit d'un filtrage, où l'on a capturé des instants marquants, touchants, surprenants, bref une sélection affective qui va au final conditionner la construction de nos souvenirs sous une facette idéalisante. D'une certaine manière, Mad Men, en revisitant avec un regard critique une période littéralement mythifiée du $\mathrm{xx}^{\mathrm{e}}$ siècle, invite par contrecoup le spectateur à une réflexion sur le décalage entre l'identité notre propre époque et la représentation que nous lui associons.

Mad Men est la transposition post-moderniste d'un mélange de nostalgie et de désillusion sur une Amérique qui se présentait comme l'écrin de tous les rêves du monde occidental de l'après-guerre. Pour le spectateur de ce début de $\mathrm{XXI}^{\mathrm{e}}$ siècle, pour qui les années 50 brillent de l'éclat du Paradis perdu dont on n'a eu de cesse de dériver depuis, la série prend un goût doux-amer lorsque qu'il apparaît que l'écrin est en réalité une boîte de Pandore.

9 «A good story is life, with the dull parts taken out. » cité dans James S. Bell, 2009, The Art Of War For Writers, Writer's Digest Books : 72. 